\title{
Inflammatory Biomarkers in Febrile Seizure: A Comprehensive Bibliometric, Review and Visualization Analysis ${ }^{\dagger}$
}

\author{
Ionela Maniu ${ }^{1,2, *}$, Raluca Costea ${ }^{2,3,4}$, George Maniu ${ }^{1}$ and Bogdan Mihai Neamtu ${ }^{2,5,6}$ \\ 1 Mathematics and Informatics Department, Research Center in Informatics and Information Technology \\ Faculty of Sciences, Lucian Blaga University of Sibiu, 550012 Sibiu, Romania; george.maniu@ulbsibiu.ro \\ 2 Pediatric Research and Telemedicine Center in Neurological Diseases, Pediatric Clinical Hospital Sibiu, \\ 550166 Sibiu, Romania; ralucacosteadr@gmail.com (R.C.); bogdan.neamtu@ulbsibiu.ro (B.M.N.) \\ 3 Pediatric Neurology Department, Pediatric Clinical Hospital Sibiu, 550166 Sibiu, Romania \\ 4 Medical Department, Faculty of Medicine, Lucian Blaga University of Sibiu, 550169 Sibiu, Romania \\ 5 Clinical Department, Faculty of Medicine, Lucian Blaga University of Sibiu, 550169 Sibiu, Romania \\ 6 Computer and Electrical Engineering Department, Faculty of Engineering, Lucian Blaga University of Sibiu, \\ 550025 Sibiu, Romania \\ * Correspondence: ionela.maniu@ulbsibiu.ro \\ + Presented at the 1st International Electronic Conference on Brain Sciences, 10-25 November 2020; \\ Available online: https://sciforum.net/conference/IECBS.
}

Citation: Maniu, I.; Costea, R.; Maniu, G.; Neamtu, B.M.

Inflammatory Biomarkers in Febrile Seizure: A Comprehensive

Bibliometric, Review and Visualization Analysis. Proceedings 2021, 71, 1. https://doi.org/10.3390/IECBS-08539

Published: 24 November 2020

Publisher's Note: MDPI stays neutral with regard to jurisdictional claims in published maps and institutional affiliations.

Copyright: (c) 2020 by the authors. Licensee MDPI, Basel, Switzerland. This article is an open access article distributed under the terms and conditions of the Creative Commons Attribution (CC BY) license (http://creativecommons.org/licenses/by/4.0/).

\begin{abstract}
Background: The associations between inflammatory markers and many diseases have been the subject of many articles and reviews. This study presents a comprehensive bibliometric, review and visualization analysis of inflammatory biomarkers (IB) in the context of febrile seizure (FS) patients. Methods: The study focused on the IB in FS using (1) bibliometric analysis-specific indicators and maps in order to analyze and present the network of authors, journals, universities and countries, (2) automated literature screening and unsupervised clustering approach for filtering and topic cluster identification. Results: We conducted a literature mining search on FS research, specifically IB in the context of FS, using automated tool VOSViewer. The presentation and discussions on the resulted bibliometric maps and topic clusters offer a comprehensive overview, status and leading trends in existing research on inflammatory biomarkers in FS. Conclusion. The analysis using bibliometric methods and review with graphical presentations can be very useful in investigating and better understanding the relationship between FS and IB.
\end{abstract}

Keywords: febrile seizures; inflammatory biomarkers; literature mining; VOSViewer; R software; clustering; visualization

\section{Introduction}

The cumulative number of research documents in many research fields, in general and particularly in the febrile seizures (FS) field, is continuously increasing. Automated, interactive, flexible tools could be used by researchers to perform systematic literature reviews and bibliometric analysis.

The current research aimed to perform a literature mining analysis on inflammatory biomarkers (IB) in the context of febrile seizure (FS). By analyzing the published documents and their citation and co-citation data, the current research themes present in inflammatory biomarkers in FS research were identified. Among the specific objectives of this analysis were: (i) to assess the range of research topics, (ii) to identify which are the inflammatory biomarkers associated with febrile seizure discussed in the published literature; (iii) to identify who has driven this research; (iv) to assess what we can learn from this research; (v) to guide investigators' potential research directions and potential collaboration partners. To our knowledge, no previous bibliometric analysis of inflammatory (bio)markers in febrile seizure research has been performed. 


\section{Materials and Methods}

In October 2020, we conducted a literature search on Web of Science Core Collection (WoS) online databases to identify scientific contributions regarding febrile seizures and their association with inflammatory biomarkers. The search strategy included the terms "febrile seizures" alone and in combination with the terms "marker" or "biomarker" and "inflamm". The asterisk was used to retrieve related/derivative words (inflammation, inflammatory, etc.). The search identified publications which contained the mentioned terms in their title or abstract or keywords. RC and BMN reviewed the pool of documents selected by the automated review to reach a consensus on the inclusion of those relevant to the topic. Discrepancies were resolved with discussion.

Characteristics of each publication identified from the search include, besides publication title, abstract and keyword, the following: authorship, document type, publication year, journal title, language, journal category, number of total citations. Data were extracted from WoS databases and exported as "tab-delimited text file" and "excel file" for additional processing.

The VOSviewer tool [1] was used to visualize and create network maps of the bibliometric indicators. Moreover, words from the title and abstracts were parsed, analyzed and visualized using this instrument.

\section{Results}

The literature search using VOSViewer resulted in 71 publications indexed in WoS, the earliest published in 1996. Contributions, in the field of febrile seizures and their association with inflammatory biomarkers, came from 114 institutions located in 18 countries/regions and were published in 579 journals.

\subsection{Distribution of Publications by Country/Region, Institution and Publication Journal}

International collaboration in the context of the analyzed studies was highlighted by constructing visualization maps of countries and institutions involved in research on inflammatory markers in febrile seizures. Collaboration analysis of countries/regions is presented in Figure 1.

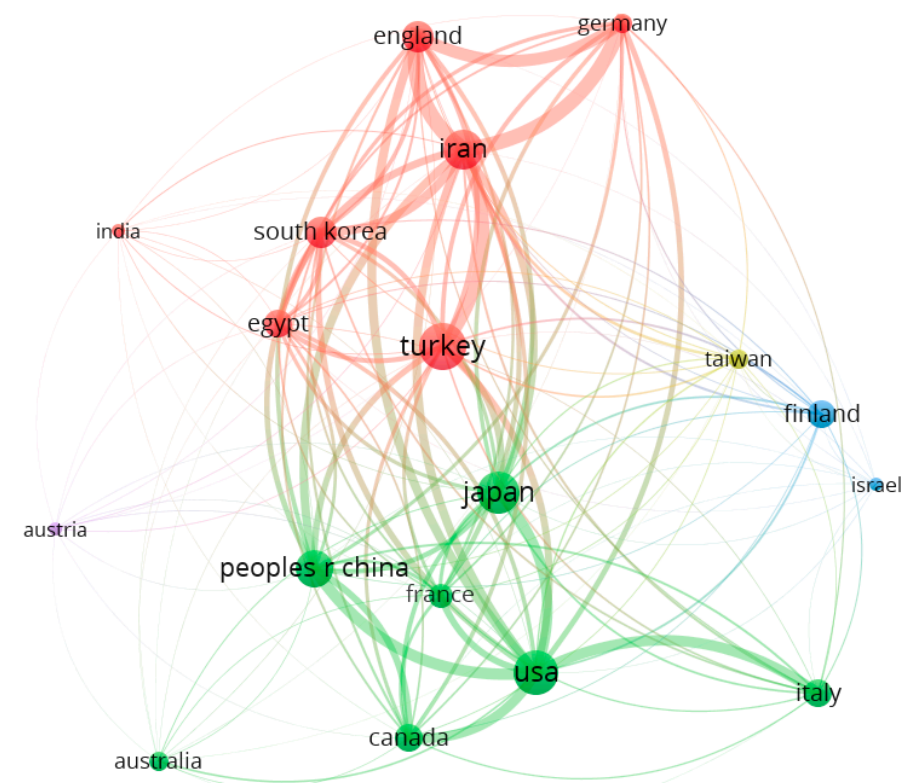

Figure 1. VOSviewer network visualization map (type of analysis: bibliographic coupling, weights-documents) of countries/regions involved in research on inflammatory markers in febrile seizures. 
Collaboration analysis of institutions is presented in Figure 2. The top five organizations in terms of produced documents are Tehran Univ. of Medical Sciences (5 publications), Mario Negri Institute for Pharmacological Research (4 publications), University of California, Irvine (UCI) (4 publications), Ehime Univ. (4 publications) and University of Social Welfare \& Rehabilitation Sciences (4 publications).

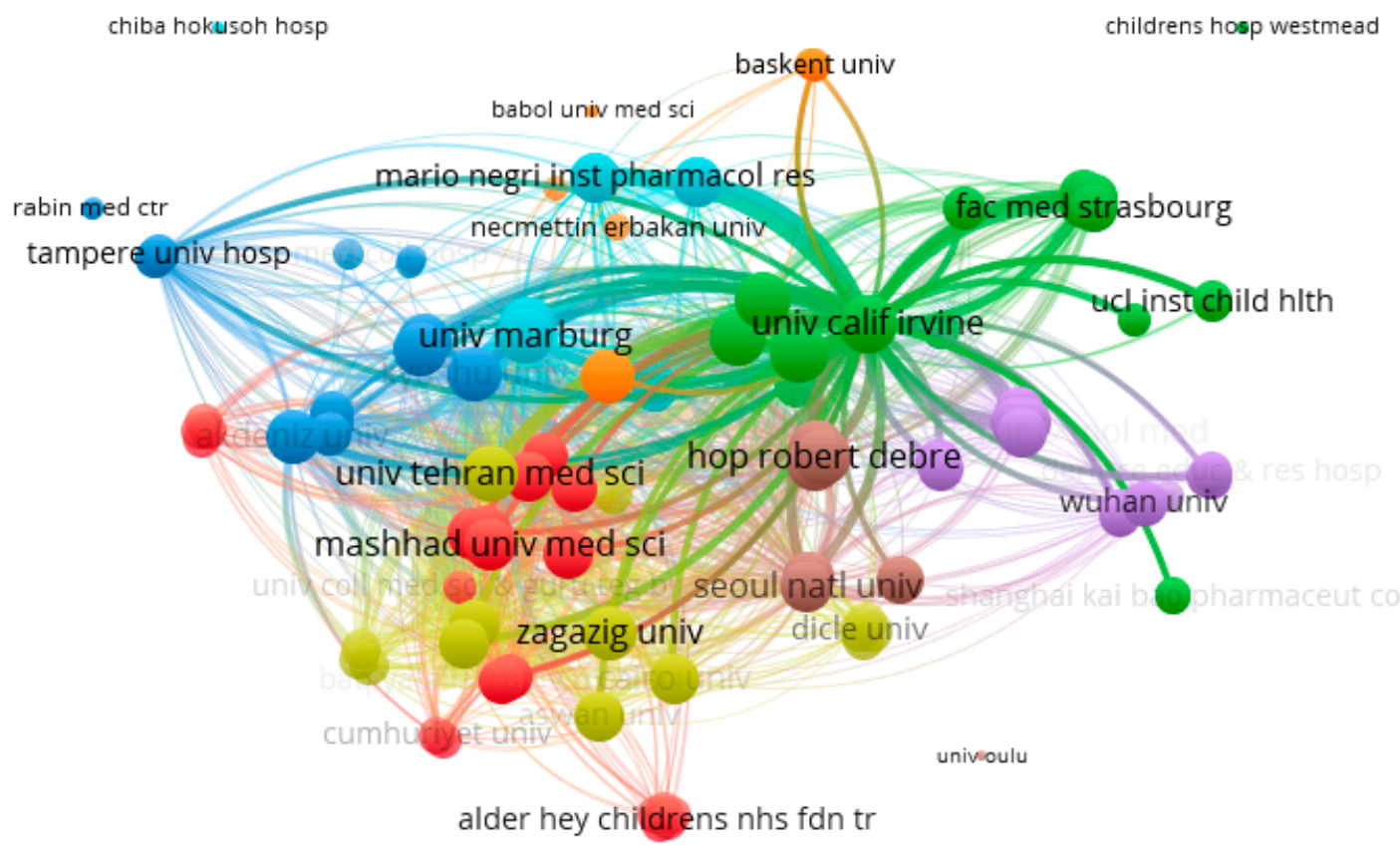

Figure 2. VOSviewer network visualization map (type of analysis: bibliographic coupling, weights - links) of institutions involved in research on inflammatory markers in febrile seizures.

The publications on inflammatory markers in febrile seizures were published in 579 academic journals. Of this research journals, 21 had at least 20 citations and are visualized in Figure 3.

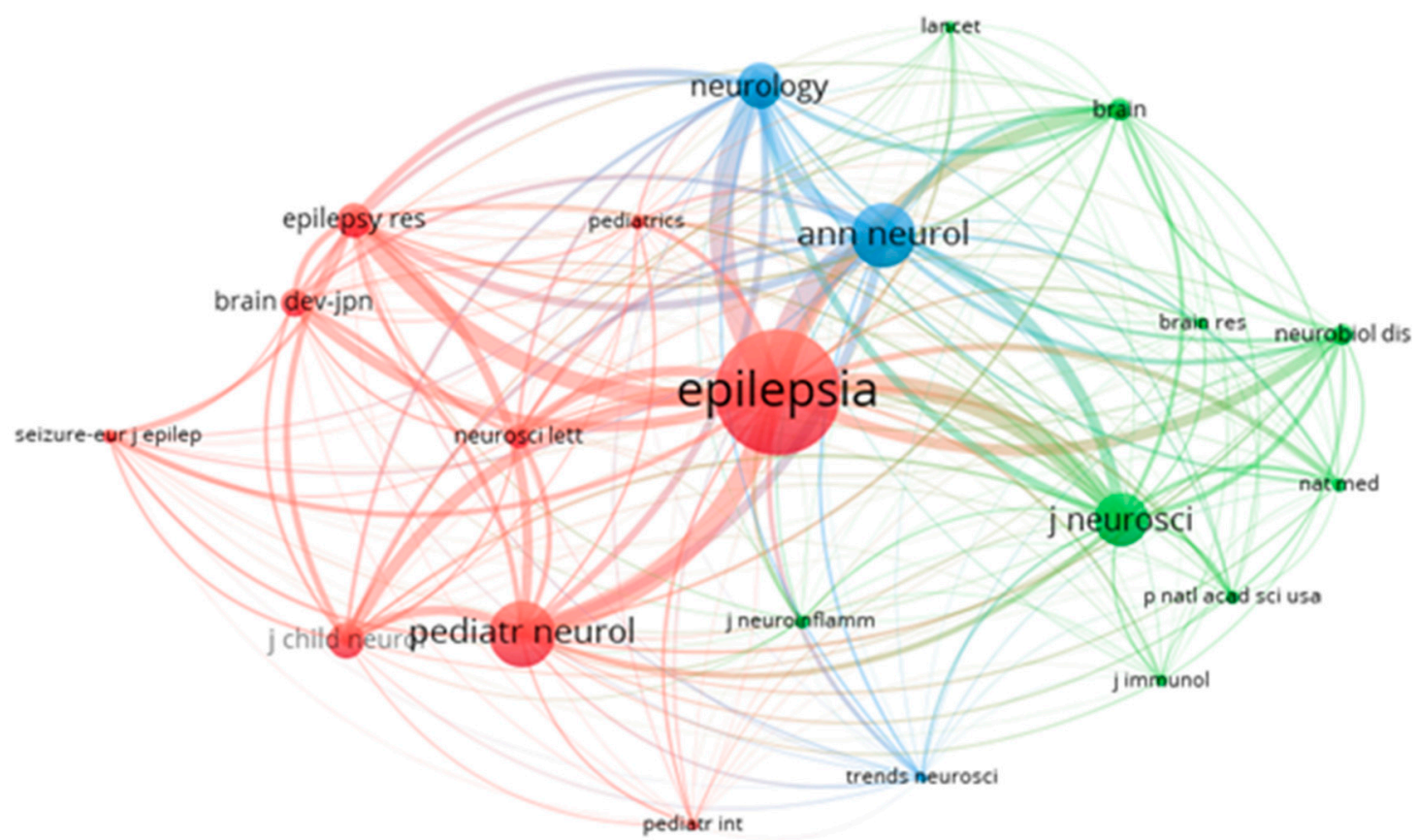

Figure 3. VOSviewer network visualization map (type of analysis: bibliographic coupling, weights - links) of journals. 


\subsection{Inflammatory Markers in Context of Febrile Seizure Research}

Indices of the inflammatory response (in the context of febrile seizures) identified by the literature mining are: (pro/anti-inflammatory) cytokines such as interleukin (IL)-1 $\beta$, IL-6, IL-8, IL10, IL-22, tumor necrosis factor (TNF)- $\alpha$ ), neutrophil-to-lymphocyte ratio (NLR), mean platelet volume (MPV), platelet count (PLT), red blood cell distribution width (RDW). They are visually represented in Figures 4 and 5.

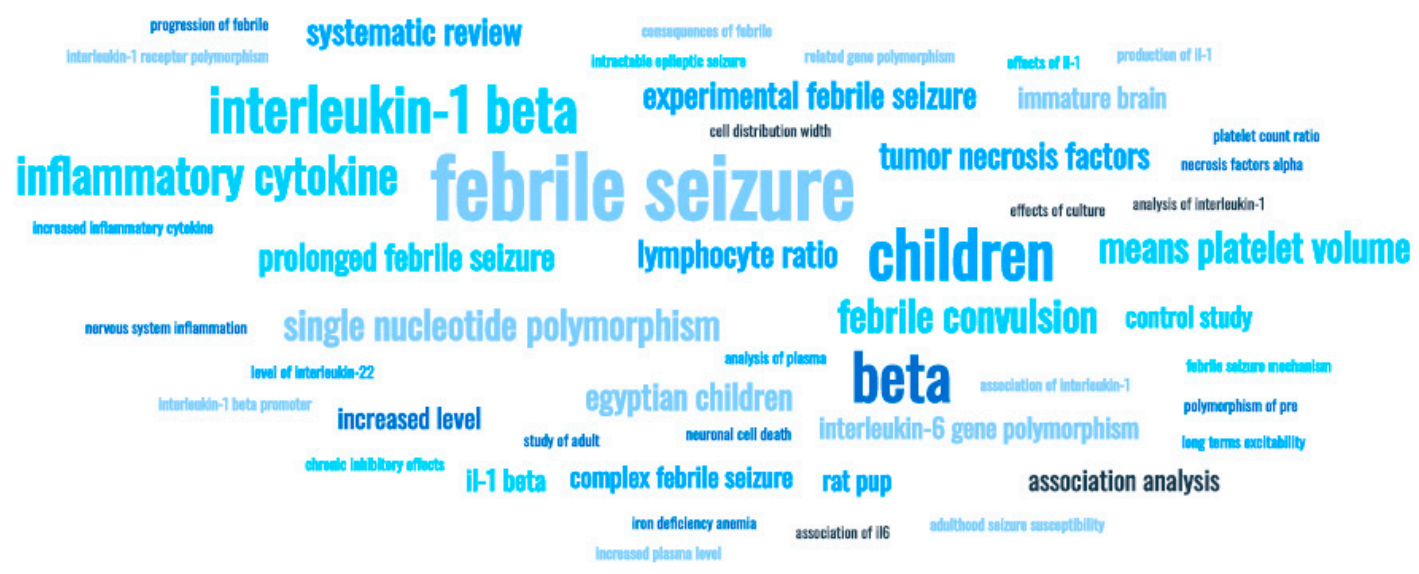

Figure 4. Inflammatory markers in context of febrile seizure research (visualization using Monkey Learn tool).

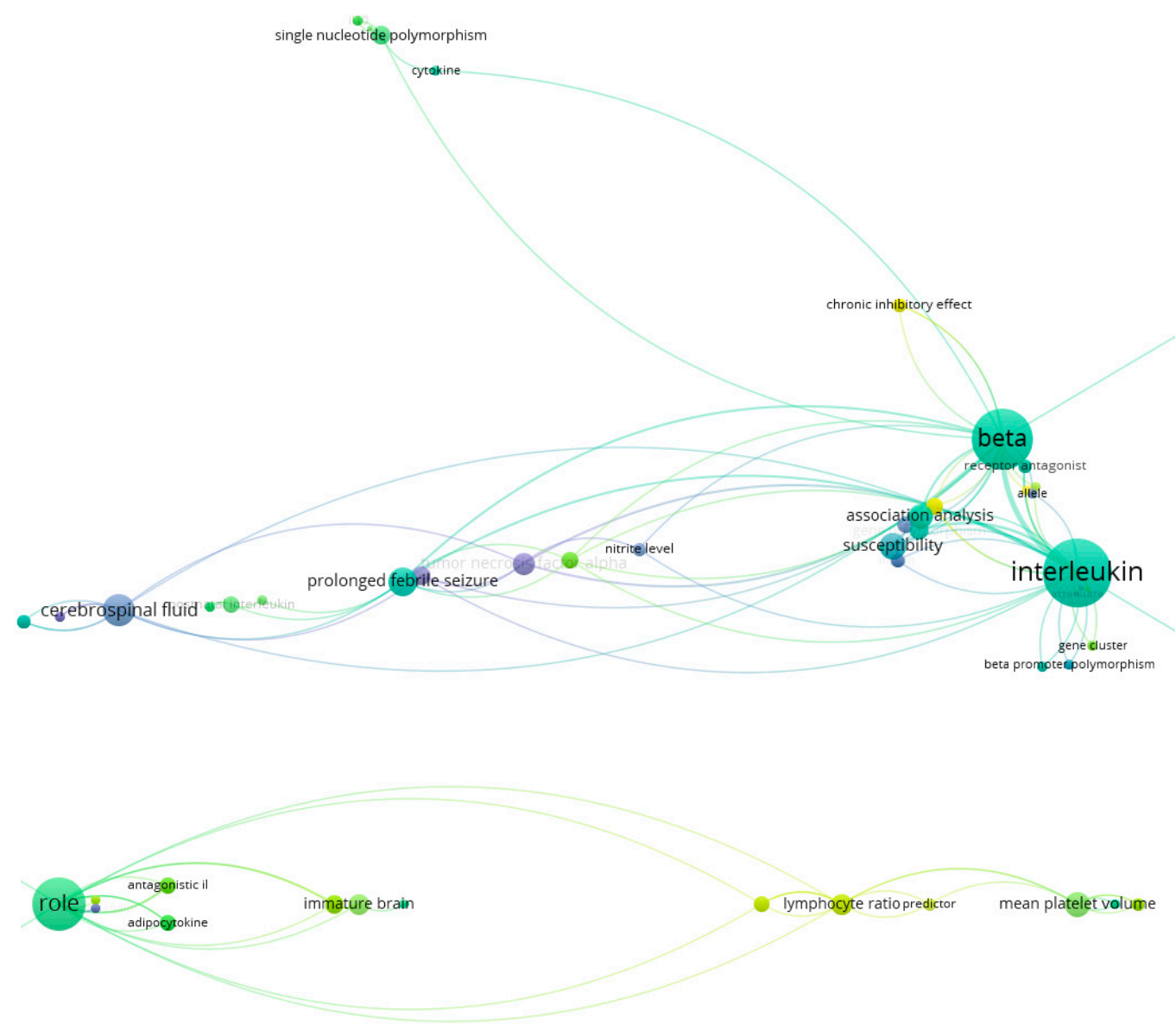

Figure 5. Inflammatory markers in context of febrile seizure research: visualization using VOSviewer. 


\section{Discussion and Conclusions}

Although the pathogenic mechanisms of febrile seizures are unclear, experimental studies demonstrate that inflammation and inflammatory mediators are the main causes and propagators of febrile seizures [2]. New trends are targeting cytokines as more sensitive, yet more expensive biomarkers, in exploring febrile seizures, as evidenced by our bibliometric tool search. Our bibliometric analysis identified little evidence of studies on common inflammatory biomarkers in febrile seizures.

The use of bibliometric analysis could guide researchers towards journals associated with the field of their research interest. Furthermore, it could provide clues and facilitate links to other research centers with the same field of interest as we identified five main institutions (Tehran Univ. of Medical Sciences, Mario Negri Institute for Pharmacological Research, University of California, Irvine (UCI), Ehime Univ., University of Social Welfare \& Rehabilitation Sciences) with activity related to inflammation and febrile seizures.

Researchers should also consider combining different bibliometric, review and metaanalysis tools [3-7] for their literature mining research.

Author Contributions: I.M. and G.M. conceived the work, performed data collection and analysis, and drafted the manuscript. R.C. and B.M.N. participated in data preprocessing and drafting of the manuscript. All authors have read and agreed to the published version of the manuscript.

Funding: This research received no external funding.

Acknowledgments: Project financed from Lucian Blaga University of Sibiu research grants LBUSIRG-2017-03. It has been conducted in the Pediatric Clinic Hospital Sibiu, within the Research and Telemedicine Center in Neurological Diseases in Children-CEFORATEN project (ID 928 SMISCSNR 13605) financed by ANCSI with the grant number 432/21.12.2012 through the Sectoral Operational Programme "Increase of Economic Competitiveness".

Conflicts of Interest: The authors declare no conflict of interest.

\section{References}

1. Van Eck, N.J.; Waltman, L. Software survey: VOSviewer, a computer program for bibliometric mapping. Scientometrics 2009, 84, 523-538, doi:10.1007/s11192-009-0146-3.

2. Dubé, C.M.; Brewster, A.L.; Richichi, C.; Zha, Q.; Baram, T.Z. Fever, febrile seizures and epilepsy. Trends Neurosci. 2007, 30, 490496.

3. Crisan, A.; Munzner, T.; Gardy, J.L.; Wren, J. Adjutant: An R-based tool to support topic discovery for systematic and literature reviews. Bioinformatics 2019, 35, 1070-1072.

4. Aria, M.; Cuccurullo, C. Bibliometrix: An R tool for comprehensive science mapping analysis. J. Informetr. 2017, 11, 959-975.

5. Westgate, M.J. Revtools: Bibliographic data visualization for evidence synthesis in R. bioArXiv 2018, doi:10.1101/262881.

6. Lee, H.; Kang, P. Identifying core topics in technology and innovation management studies: A topic model approach. J. Technol. Transf. 2018, 43, 1291-1317.

7. Crisan, A.; Gardy, J.L.; Munzner, T. A systematic method for surveying data visualizations and a resulting genomic epidemiology visualization typology: GEViT. Bioinformatics 2019, 35, 1668-1676, doi:10.1093/bioinformatics/bty832. 